\title{
WEAK SHOCK WAVES FOR THE GENERAL DISCRETE VELOCITY MODEL OF THE BOLTZMANN EQUATION*
}

\author{
NICLAS BERNHOFF ${ }^{\dagger}$ AND ALEXANDER BOBYLEV $\ddagger$
}

\begin{abstract}
We study the shock wave problem for the general discrete velocity model (DVM), with an arbitrary finite number of velocities. In this case the discrete Boltzmann equation becomes a system of ordinary differential equations (dynamical system). Then the shock waves can be seen as heteroclinic orbits connecting two singular points (Maxwellians). In this paper we give a constructive proof for the existence of solutions in the case of weak shocks.

We assume that a given Maxwellian is approached at infinity, and consider shock speeds close to a typical speed, corresponding to the sound speed in the continuous case. The existence of a non-negative locally unique (up to a shift in the independent variable) bounded solution is proved by using contraction mapping arguments (after a suitable decomposition of the system). This solution is shown to tend to a Maxwellian at minus infinity.

Existence of weak shock wave solutions for DVMs was proved by Bose, Illner and Ukai in 1998. In this paper, we give a constructive, more straightforward, proof that suits the discrete case. Our approach is based on earlier results by the authors on the main characteristics (dimensions of corresponding stable, unstable and center manifolds) for singular points of general dynamical systems of the same type as in the shock wave problem for DVMs.
\end{abstract}

The same approach can also be applied for DVMs for mixtures.

Key words. Boltzmann equation, discrete velocity models, shock waves

AMS subject classifications. $82 \mathrm{C} 40,76 \mathrm{P} 05$

\section{Introduction}

We are concerned with the existence of shock wave solutions $f=f\left(x^{1}, \xi, t\right)=$ $F\left(x^{1}-c t, \xi\right)$, of the Boltzmann equation

$$
\frac{\partial f}{\partial t}+\xi \cdot \nabla_{\mathbf{x}} f=Q(f, f) .
$$

Here $\mathbf{x}=\left(x^{1}, \ldots, x^{d}\right) \in \mathbb{R}^{d}, \xi=\left(\xi^{1}, \ldots, \xi^{d}\right) \in \mathbb{R}^{d}$ and $t \in \mathbb{R}_{+}$denote position, velocity and time respectively. Furthermore, $c>c_{0}$ denotes the speed of the wave, where $c_{0}$ is the speed of sound. The solutions are assumed to approach two given Maxwellians $M_{ \pm}=$ $\frac{\rho_{ \pm}}{\left(2 \pi T_{ \pm}\right)^{d / 2}} e^{-\left|\xi-\mathbf{u}_{ \pm}\right|^{2} /\left(2 T_{ \pm}\right)}(\rho, \mathbf{u}$ and $T$ denote density, bulk velocity and temperature respectively) as $x \rightarrow \pm \infty$, which are related through the Rankine-Hugoniot conditions.

The (shock wave) problem is to find a solution $F=F(y, \xi)\left(y=x^{1}-c t\right)$ of the equation

$$
\left(\xi^{1}-c\right) \frac{\partial F}{\partial y}=Q(F, F)
$$

such that

$$
f \rightarrow M_{ \pm} \text {as } y \rightarrow \pm \infty
$$

${ }^{*}$ Received: April 3, 2007; accepted (in revised version): July 31, 2007. Communicated by Francois Bouchut.

${ }^{\dagger}$ Department of Mathematics, Karlstad University, 65188 Karlstad, Sweden (niclas.bernhoff@ kau.se).

${ }^{\ddagger}$ Department of Mathematics, Karlstad University, 65188 Karlstad, Sweden (alexander.bobylev@ kau.se). 
In this paper, we consider the shock wave problem (1.1), (1.2) for the general discrete velocity model (DVM) (the discrete Boltzmann equation) $[5,10]$. We allow the velocity variable to take values only from a finite subset $\mathrm{V}$ of $\mathbb{R}^{d}$, i.e., $\xi \in \mathrm{V}=$ $\left\{\xi_{1}, \ldots, \xi_{n}\right\} \subset \mathbb{R}^{d}$, where $n$ is an arbitrary natural number.

We obtain, from Equation (1.1), a system of ODEs (dynamical system)

$$
\left(\xi_{i}^{1}-c\right) \frac{d F_{i}}{d y}=Q_{i}(F, F), i=1, \ldots, n, c \in \mathbb{R},
$$

where $F=\left(F_{1}, \ldots, F_{n}\right)$, with $F_{i}=F_{i}(y)=F\left(y, \xi_{i}\right), i=1, \ldots, n$. The collision operator $Q=\left(Q_{1}, \ldots, Q_{n}\right)$ is given by

$$
Q_{i}(F, G)=\frac{1}{2} \sum_{j, k, l=1}^{n} \Gamma_{i j}^{k l}\left(F_{k} G_{l}+G_{k} F_{l}-F_{i} G_{j}-G_{i} F_{j}\right), i=1, \ldots, n,
$$

where it is assumed that the collision coefficients $\Gamma_{i j}^{k l}$ satisfy the relations $\Gamma_{i j}^{k l}=\Gamma_{j i}^{k l}=$ $\Gamma_{k l}^{i j} \geq 0$, with equality unless

$$
\xi_{i}+\xi_{j}=\xi_{k}+\xi_{l} \text { and }\left|\xi_{i}\right|^{2}+\left|\xi_{j}\right|^{2}=\left|\xi_{k}\right|^{2}+\left|\xi_{l}\right|^{2} .
$$

$Q(F, G)$ is a bounded bilinear operator symmetric in arguments. Hence, there exists a constant $C$, such that

$$
|Q(F, G)| \leq C|F||G| \text {, for all } F, G \in \mathbb{R}^{n},
$$

where $|F|$ is the usual Euclidean norm of $F \in \mathbb{R}^{n}$.

For normal (only with physical collision invariants) DVMs the collision invariants (i.e., all $\phi=\left(\phi_{1}, \ldots, \phi_{n}\right)$ such that $\phi_{i}+\phi_{j}=\phi_{k}+\phi_{l}$ if $\left.\Gamma_{i j}^{k l} \neq 0\right)$ are of the form

$$
\phi=\left(\phi_{1}, \ldots, \phi_{n}\right), \phi_{i}=\alpha+\beta \cdot \xi_{i}+\gamma\left|\xi_{i}\right|^{2}, \quad \alpha, \gamma \in \mathbb{R}, \beta \in \mathbb{R}^{d},
$$

and the Maxwellians (positive vectors $M=\left(M_{1}, \ldots, M_{n}\right), M_{1}, \ldots, M_{n}>0$, such that $Q(M, M)=0)$ are of the form

$$
M=\left(M_{1}, \ldots, M_{n}\right), M_{i}=A e^{\beta \cdot \xi_{i}+\gamma\left|\xi_{i}\right|^{2}}, \text { with } A=e^{\alpha}>0, \quad \alpha, \gamma \in \mathbb{R}, \beta \in \mathbb{R}^{d} .
$$

We denote by $\left\{\phi_{1}, \ldots, \phi_{p}\right\}$ ( $p=d+2$ for normal DVMs) a basis for the vector space of collision invariants (note that here and below $\phi_{i}$ denotes a collision invariant, while above $\phi_{i}$ denotes the $i$ th component of the collision invariant $\phi$ ). Then

$$
\left\langle\phi_{i}, Q(f, f)\right\rangle=0 \text { for } i=1, \ldots, p .
$$

Here and below $\langle\cdot, \cdot\rangle$ denotes the Euclidean scalar product and we denote $\langle\cdot, \cdot\rangle_{E}=\langle\cdot, E \cdot\rangle$ for symmetric matrices $E$.

The shock wave problem for the discrete Boltzmann equation reads

$$
(B-c I) \frac{d F}{d y}=Q(F, F), \text { where } F \rightarrow M_{ \pm} \text {as } y \rightarrow \pm \infty,
$$

where $B$ is the diagonal matrix

$$
B=\operatorname{diag}\left(\xi_{1}^{1}, \ldots, \xi_{n}^{1}\right)
$$


Note that shock waves for the discrete Boltzmann equation can be seen as heteroclinic orbits connecting two singular points (which are Maxwellians for DVMs). If we multiply Equation (1.5) scalarly by $\phi_{i}, 1 \leq i \leq p$, and integrate over $\mathbb{R}$, then we obtain that the Maxwellians $M_{-}$and $M_{+}$must fulfill the Rankine-Hugoniot conditions

$$
\left\langle M_{+}, \phi_{i}\right\rangle_{B-c I}=\left\langle M_{-}, \phi_{i}\right\rangle_{B-c I}, i=1, \ldots, p .
$$

The rest of this paper is organized as follows. In Section 2, we state under which assumptions our results are obtained and present the main results. In Section 3 we fix the Maxwellian $M_{+}$approached at infinity, and consider shock speeds close to a typical speed $c_{0}$ (corresponding to the speed of sound in the continuous case). We expand around the Maxwellian $M_{+}$, make a transformation and obtain a new system of ODEs. In Sections 4 and 5 the existence of a non-negative locally unique (up to a shift in the independent variable) bounded solution is proved by using contraction mapping arguments. In Section 6 we show that this solution tends to a Maxwellian at minus infinity, using arguments used in [7]. Finally, in Section 7 we prove a lemma used in Section 4.

Some of our results can probably be deduced from the general theory of ODEs related to bifurcations of saddle points [1]. Such an approach in a more abstract setting was used for general hyperbolic systems in [9]. It is not easy to determine whether the conditions of [9] hold for our Equation (1.3). The difficulty is that we do not have the explicit relations between conservative quantities (density, energy, and momentum) and parameters of equilibrium (Maxwellian) distributions for general DVMs. Paradoxically, such (very simple) explicit relations exist in the continuum limit. Therefore equations of hydrodynamics for the Boltzmann equation are, in a sense, simpler than similar equations for the general DVM. On the other hand, very general results of [9] can be applied to various versions of moment equations, whereas our approach is based on specific properties of DVMs. We prefer, however, to use a straightforward approach, which clarifies many details of this specific problem.

\section{Assumptions and main results}

We make the following assumptions in our DVMs.

1. There is a number $c_{0}$ ("speed of sound"), with the following properties:

[i] $\operatorname{rank}(K)=p-1$, where $K$ is the $p \times p$ matrix with the elements (here and below multiplication of two vectors in $\mathbb{R}^{n}$ means to multiply corresponding components to obtain a new vector in $\mathbb{R}^{n}$ )

$$
k_{i j}=\left\langle M_{+} \phi_{i}, \phi_{j}\right\rangle_{B-c_{0} I} .
$$

The rank of $K$ is independent of the choice of the basis $\left\{\phi_{1}, \ldots, \phi_{p}\right\}$. In other words, there is a unique (up to its sign) vector $\phi_{\perp}$ in $\operatorname{span}\left(\phi_{1}, \ldots, \phi_{p}\right)$, such that $\left\langle M_{+} \phi_{\perp}, \phi_{\perp}\right\rangle=1$ and

$$
\left\langle M_{+} \phi_{\perp}, \phi\right\rangle_{B-c_{0} I}=0 \text { for all } \phi \in \operatorname{span}\left(\phi_{1}, \ldots, \phi_{p}\right) .
$$

[ii] $c_{0} \neq \xi_{i}^{1}$ for $i=1, \ldots, n$, or, equivalently, $\operatorname{det}\left(B-c_{0} I\right) \neq 0$.

2. The vector(s) $\phi_{\perp}$ fulfilling Equation (2.1), also satisfy $\left\langle M_{+} \phi_{\perp}, \phi_{\perp}^{2}\right\rangle_{B-c_{0} I} \neq 0$. We choose the sign of the vector $\phi_{\perp}$ such that $\left\langle M_{+} \phi_{\perp}, \phi_{\perp}^{2}\right\rangle_{B-c_{0} I}>0$.

Remark 2.1. Let $M_{+}$be a Maxwellian with zero bulk velocity $(\mathbf{u}=\mathbf{0})$. Then, for the "continuous" Boltzmann equation, $M_{+}=\frac{\rho}{(2 \pi T)^{d / 2}} e^{-|\xi|^{2} /(2 T)}$. In this case (see [8]), 
with $d=3, c_{0}= \pm \sqrt{\frac{5 T}{3}}$ (note that Assumption 1 [ii] never is fulfilled in the continuous case), $\phi_{\perp}=\frac{1}{\sqrt{2 \rho T}}\left(\xi^{1} \pm \frac{|\xi|^{2}}{\sqrt{15 T}}\right)$ and $\left\langle M_{+} \phi_{\perp}, \phi_{\perp}^{2}\right\rangle_{B-c_{0} I}=\frac{2}{3} \sqrt{\frac{2 T}{\rho}}>0$.

REMARK 2.2. Assume that we have an axially symmetric normal model (i.e., if $\left(\xi^{1}, \ldots, \xi^{d}\right) \in \mathrm{V}$, then $\left.\left( \pm \xi^{1}, \ldots, \pm \xi^{d}\right) \in \mathrm{V}\right)$. Let $M=A e^{\gamma|\xi|^{2}}$ and assume that the collision invariants

$$
\left\{\begin{array}{l}
\phi_{1}=(1, \ldots, 1) \\
\phi_{i+1}=\left(\xi_{1}^{i}, \ldots, \xi_{n}^{i}\right), i=1, \ldots, d \\
\phi_{d+2}=\left(\left|\xi_{1}\right|^{2}, \ldots,\left|\xi_{n}\right|^{2}\right)
\end{array}\right.
$$

are linearly independent. Then [2]

$$
\begin{gathered}
c_{0}=c_{ \pm}= \pm \sqrt{\frac{\chi_{1} \chi_{4}^{2}+\chi_{2}^{2} \chi_{5}-2 \chi_{2} \chi_{3} \chi_{4}}{\chi_{2}\left(\chi_{1} \chi_{5}-\chi_{3}^{2}\right)}}, \text { where } \\
\chi_{1}=\left\langle\phi_{1}, M \phi_{1}\right\rangle, \chi_{2}=\left\langle\phi_{2}, M \phi_{2}\right\rangle, \chi_{3}=\left\langle\phi_{1}, M \phi_{d+2}\right\rangle, \chi_{4}=\left\langle\phi_{2}, M \phi_{d+2}\right\rangle_{B} \\
\text { and } \chi_{5}=\left\langle\phi_{d+2}, M \phi_{d+2}\right\rangle .
\end{gathered}
$$

We assume that Assumptions 1 and 2 are fulfilled and denote

$$
\|h\|=\|h(y)\|=\sup _{y \in \mathbb{R}}|h(y)|
$$

for any bounded (vector or scalar) function $h(y): \mathbb{R} \rightarrow \mathbb{R}^{k}$, where $k$ is a positive integer.

A proof for existence of weak shock wave solutions for DVMs was already presented in 1998 by Bose, Illner and Ukai [4]. In their technical proof Bose et al. are following the lines of the pioneering work for the continuous Boltzmann equation by Caflisch and Nicolaenko [6] (for more resent research in the continuous case see [13]).

In this work, we follow a more straightforward way, suiting the discrete case. We use results by the authors [3] on the main characteristics (dimensions of corresponding stable, unstable and center manifolds) for singular points of general dynamical systems of the same type as in the shock wave problem for DVMs. Our assumptions differ a little from the ones made in the paper by Bose, Illner and Ukai [4]. Assumption 1 i) in this paper corresponds to assumption [H1] (i) in [4], and also Assumption 1 ii) is assumed in [4]. However, instead of transcritical bifurcation at $c=c_{0}$ (see assumption [H1] (ii) in [4]), we additionally assume Assumption 2. While the assumption of transcritical bifurcation at $c=c_{0}$ produces the "other" Maxwellian $\left(M_{-}\right.$in our case, see Theorem 2.1 below, and $M_{+}$in [4]) in a natural way, we obtain the second Maxwellian as a limiting case of our solution at minus infinity or more directly by an iteration process (see Section 6). We want to stress that our proof is constructive, and that it can also (at least implicitly) be shown how close to the typical speed $c_{0}$, the shock speed must be for our results to be valid.

THEOREM 2.1. For any given positive Maxwellian $M_{+}$, there exists a family of Maxwellians $M_{-}=M_{-}(\varepsilon)$ and shock speeds $c=c(\varepsilon)=c_{0}+\varepsilon$, such that the shock wave problem (1.5) has a non-negative locally unique (with respect to the norm $\|\cdot\|$ and up to a shift in the independent variable) non-trivial bounded solution for each sufficiently small $\varepsilon>0$. Furthermore, $M_{-}$is determined by $M_{+}$and $c$. 
REMARK 2.3. The arguments in this paper can be changed, so that we can interchange $M_{-}$and $M_{+}$in Theorem 2.1 (with $\varepsilon<0$ ).

REMARK 2.4. The approach of this paper can also be applied to obtain similar results for the discrete Boltzmann equation for mixtures.

\section{Transformation of the problem}

We consider

$$
(B-c I) \frac{d F}{d y}=Q(F, F), \text { where } F \rightarrow M_{+} \text {as } y \rightarrow \infty .
$$

We first prove the following theorem.

TheOREM 3.1. For any given positive Maxwellian $M_{+}$, there exists a family of shock numbers $c=c(\varepsilon)$, such that the problem (3.1) has a non-negative locally unique (with respect to the norm $\|\cdot\|$ and up to a shift in the independent variable) non-trivial bounded solution, for each sufficiently small $\varepsilon>0$.

Then arguments in [7] can be used to show that the solution tends to a Maxwellian at minus infinity (see Section 6 below).

We denote

$$
F=M+M^{1 / 2} h, \text { with } M=M_{+},
$$

and obtain

$$
(B-c I) \frac{d h}{d y}+L h=S(h, h), \text { where } h \rightarrow 0 \text { as } y \rightarrow \infty,
$$

with

$$
L h=-2 M^{-1 / 2} Q\left(M, M^{1 / 2} h\right) \text { and } S(g, h)=M^{-1 / 2} Q\left(M^{1 / 2} g, M^{1 / 2} h\right) .
$$

The linear operator ( $n \times n$ matrix) $L$ is symmetric and semi-positive (i.e., $\langle h, h\rangle_{L} \geq 0$ for all $h \in \mathbb{R}^{n}$ ) and has the null-space

$$
N(L)=\operatorname{span}\left(M^{1 / 2} \phi_{1}, \ldots, M^{1 / 2} \phi_{p}\right)=\operatorname{span}\left(e_{1}, \ldots, e_{p}\right),
$$

where $\left\{e_{1}, \ldots, e_{p}\right\}$ can be chosen such that

$$
\left\langle e_{i}, e_{j}\right\rangle=\delta_{i j} \text { and }\left\langle e_{i}, e_{j}\right\rangle_{B-c I}=\left(\gamma_{i}-c\right) \delta_{i j} \text {, with } \gamma_{i}=\left\langle e_{i}, e_{i}\right\rangle_{B} .
$$

The quadratic part $S(h, h)$ is orthogonal to $N(L)$ (i.e., $\langle\phi, S(h, h)\rangle=0$ if $\phi \in N(L)$ ).

By Assumption 1 [i], there is a number $c=c_{0}$, such that (after possible renumbering)

$$
\gamma_{p}=c_{0} \text { and } \gamma_{i} \neq c_{0} \text { for } i=1, \ldots, p-1 .
$$

We study Equation (3.2) for

$$
c=c_{0}+\varepsilon, 0<\varepsilon \leq s,
$$

where $s$ is chosen such that

$$
\operatorname{det}(B-c I) \neq 0 \text { and } \gamma_{i} \neq c, i=1, \ldots, p \text {, if } 0<\varepsilon \leq s .
$$


Clearly, (for a finite number $n$ ) such a number $s$ exists by Assumption 1.

Then Equation (3.2) are equivalent with the system

$$
\frac{d h}{d y}+(B-c I)^{-1} L h=(B-c I)^{-1} S(h, h) .
$$

We now formulate a result on the characterization of corresponding linearized system [3]. Let $n^{ \pm}$, with $n^{+}+n^{-}=n$, and $m^{ \pm}$, denote the numbers of the positive and negative eigenvalues of the matrices $B-c I$ and $(B-c I)^{-1} L$ respectively. Moreover, let $k^{+}, k^{-}$, and $l$ be the numbers of positive, negative, and zero eigenvalues of the $p \times p$ matrix $K$, with entries $k_{i j}=\left\langle y_{i}, y_{j}\right\rangle_{B-c I}$, such that $N(L)=\operatorname{span}\left(y_{1}, \ldots, y_{p}\right)$. Then $m^{ \pm}=n^{ \pm}-k^{ \pm}-l$, and the matrix $(B-c I)^{-1} L$ is diagonalizable if and only if $l=0$. This result is independent on the choice of the basis $\left\{y_{1}, \ldots, y_{p}\right\}$ of $N(L)$. In particular, it is true for $\left\{y_{1}, \ldots, y_{p}\right\}=\left\{e_{1}, \ldots, e_{p}\right\}$.

Remark 3.1. Equation (3.3)-(3.5), imply that $l=1$ if $\varepsilon=0$, and $l=0$ if $0<\varepsilon \leq s$, while $n^{+}$and $k^{+}$do not change for $0 \leq \varepsilon \leq s$. Therefore, $(B-c I)^{-1} L$ has exactly one more positive eigenvalue, for $0<\varepsilon \leq s$, than for $\varepsilon=0$.

The matrix $(B-c I)^{-1} L$ has (for $0<\varepsilon \leq s$ ) exactly $n-p$ non-zero (real) eigenvalues. Moreover, there is a basis $\left\{u_{0}, \ldots, u_{m}\right\}$, with $m=n-p-1$, of $\operatorname{Im}\left((B-c I)^{-1} L\right)$, such that [3]

$$
\begin{gathered}
(B-c I)^{-1} L u_{i}=\lambda_{i} u_{i}, \lambda_{i} \neq 0,\left\langle u_{i}, u_{j}\right\rangle_{B-c I}=\lambda_{i} \delta_{i j}, \\
u_{i}=(B-c I)^{-1} L^{1 / 2} w_{i},\left\langle w_{i}, w_{j}\right\rangle=\delta_{i j}, i, j=0, \ldots, m .
\end{gathered}
$$
0 .

We choose $u_{0}$, such that $\lambda_{0}$ is the minimal positive eigenvalue and $\left\langle u_{0}, M^{1 / 2} \phi_{\perp}\right\rangle \geq$

REMARK 3.2. The relation

$$
\operatorname{det}\left((B-c I)^{-1} L-\lambda I\right)=0 \Leftrightarrow \operatorname{det}\left(L-\lambda\left(B-c_{0} I-\varepsilon I\right)\right)=0
$$

implies that the real eigenvalues of $(B-c I)^{-1} L$ are continuous in $\varepsilon$. In fact,

$$
\begin{aligned}
\operatorname{det}\left(L-\lambda\left(B-c_{0} I-\varepsilon I\right)\right) & =s_{n}(\varepsilon) \lambda^{n}+\ldots+s_{p}(\varepsilon) \lambda^{p} \\
& =s_{n}(\varepsilon) \lambda^{p} \prod_{i=0}^{m}\left(\lambda-\lambda_{i}(\varepsilon)\right),
\end{aligned}
$$

where $s_{p}(\varepsilon), \ldots, s_{n}(\varepsilon)$ are polynomials in $\varepsilon$, such that $s_{n}(\varepsilon)=\operatorname{det}(B-c I) \neq 0, s_{p}(0)=0$ $\left(s_{p}(\varepsilon) \neq 0\right.$ if $\left.0<\varepsilon \leq s\right)$ and $s_{p+1}(0) \neq 0$.

Hence,

$$
0<\lambda_{0}<\lambda_{i}, i=1, \ldots, m,
$$

if $\varepsilon$ is small enough. Moreover, by Equation (3.7) and Assumption 1 we can conclude that

$$
\lambda_{0}=O(\varepsilon) \text { and } \lambda_{i}=O(1), i=1, \ldots, m, \text { as } \varepsilon \rightarrow 0^{+} .
$$

Furthermore, by the implicit function theorem, the eigenvalue $\lambda_{0}=\lambda_{0}(\varepsilon)$ is a $C^{1}$ function (in an open neighborhood of $\varepsilon=0$ ), with the first derivative

$$
\frac{d \lambda_{0}}{d \varepsilon}(\varepsilon)=\frac{\frac{d s_{n}}{d \varepsilon}(\varepsilon) \lambda_{0}^{n}+\cdots+\frac{d s_{p}}{d \varepsilon}(\varepsilon) \lambda_{0}^{p}}{n s_{n}(\varepsilon) \lambda_{0}^{n-1}+\cdots+p s_{p}(\varepsilon) \lambda_{0}^{p-1}} .
$$


In particular,

$$
\frac{d \lambda_{0}}{d \varepsilon}(0)=\frac{\frac{d s_{p}}{d \varepsilon}(0)}{(p+1) s_{p+1}(0)}
$$

The smallness of $\lambda_{0}$, compared to the other eigenvalues, is essential in the proof.

These results can also be deduced by perturbation theory [12].

We denote

$$
h=\sum_{i=0}^{m} x_{i} u_{i}, \text { where } x_{i}=x_{i}(y)=\frac{1}{\lambda_{i}}\left\langle h, u_{i}\right\rangle_{B-c I} .
$$

Then,

$$
\frac{d x_{i}}{d y}+\lambda_{i} x_{i}=g_{i}(X, X)
$$

where $X=\left(x_{0}, \ldots, x_{m}\right), g_{i}=g_{i}(X, Y)=\sum_{j, k=0}^{m} x_{j} y_{k} g_{j k}^{i}, i=0, \ldots, m$,

with $g_{j k}^{i}=\frac{1}{\lambda_{i}}\left\langle u_{i}, S\left(u_{j}, u_{k}\right)\right\rangle=\left\langle L^{-1 / 2} w_{i}, S\left((B-c I)^{-1} L^{1 / 2} w_{j},(B-c I)^{-1} L^{1 / 2} w_{k}\right)\right\rangle$.

We denote by $\widehat{g}_{i}$ the symmetric $(m+1) \times(m+1)$ matrix with entries

$$
\left(\widehat{g}_{i}\right)_{j+1, k+1}=g_{j k}^{i}, 0 \leq j, k \leq m,
$$

and by $\mathcal{G}_{i}>0$ the maximum of the absolute values of the eigenvalues of the matrix $\widehat{g}_{i}$, or, equivalently, $\mathcal{G}_{i}=\sup _{|X|=1}\left|\widehat{g}_{i} X\right|$. Then

$$
g_{i}(X, Y)=\left\langle X, \widehat{g}_{i} Y\right\rangle \text { and }\left|g_{i}(X, Y)\right| \leq \mathcal{G}_{i}|X||Y|, \text { for } i=0, \ldots, m
$$

Let $\sigma_{1}, \ldots, \sigma_{m+1}$ denote the non-zero (i.e., positive) eigenvalues of the $n \times n$ matrix $L$. Then

$$
\left|g_{j k}^{i}\right| \leq \frac{C M_{\max } \sigma_{\max }}{b_{\min }^{2} \sqrt{M_{\min } \sigma_{\min }}}, 0 \leq i, j, k \leq m,
$$

with $C$ from Equation (1.4) and

$$
\begin{gathered}
\sigma_{\max }=\max _{1 \leq \alpha \leq m+1}\left(\sigma_{\alpha}\right), \sigma_{\min }=\min _{1 \leq \alpha \leq m+1}\left(\sigma_{\alpha}\right), b_{\min }=\min _{1 \leq \alpha \leq n}\left|\xi_{\alpha}^{1}-c\right|, \\
M_{\min }=\min _{1 \leq i \leq n}\left(M_{i}\right) \text { and } M_{\max }=\max _{1 \leq i \leq n}\left(M_{i}\right) .
\end{gathered}
$$

Hence,

$$
\mathcal{G}_{i} \leq \frac{C M_{\max } \sigma_{\max }(m+1)}{b_{\min }^{2} \sqrt{M_{\min } \sigma_{\min }}}, i=1, \ldots, m .
$$

It is clear that $x_{0}=x_{0}(y)$ plays a special role for small values of the minimal positive eigenvalue $\lambda_{0}$ (and therefore also for small $\varepsilon$ ). We assume that $x_{0} \neq 0$ and substitute

$$
\left\{\begin{array}{l}
x_{0}(y)=\lambda_{0} x(t) \\
x_{i}(y)=\lambda_{0} x(t) z_{i}(t)
\end{array} \text { with } t=\lambda_{0} y, \text { for } i=1, \ldots, m\right.
$$


Denoting

$$
\begin{gathered}
Z=\left(1, z_{1}, \ldots, z_{m}\right), z=\left(z_{1}, \ldots, z_{m}\right), \theta=\theta(z)=g_{0}(Z, Z) \\
\text { and } \mu_{i}=\frac{\lambda_{0}}{\lambda_{i}-\lambda_{0}}, i=1, \ldots, m,
\end{gathered}
$$

we obtain

$$
\left\{\begin{array}{l}
\frac{d x}{d t}+x=x^{2} g_{0}(Z, Z) \\
x \frac{d z_{i}}{d t}+\frac{d x}{d t} z_{i}+\frac{\lambda_{i}}{\lambda_{0}} x z_{i}=x^{2} g_{i}(Z, Z)
\end{array} i=1, \ldots, m\right.
$$

or, equivalently,

$$
\left\{\begin{array}{l}
\frac{d x}{d t}+x=x^{2} \theta(z) \\
\frac{d z_{i}}{d t}+\frac{1}{\mu_{i}} z_{i}=x\left(g_{i}(Z, Z)-z_{i} \theta(z)\right)
\end{array} \quad i=1, \ldots, m\right.
$$

\section{Existence of a non-trivial bounded solution}

From the first equation in Equation (3.12) we obtain

$$
\frac{d}{d t}\left(e^{-t} \frac{1}{x}\right)=-e^{-t} \theta(z)
$$

We note that $x(t) \rightarrow 0$ as $t \rightarrow \infty$. Moreover, if Equation (3.12) has a bounded solution (with $z$ bounded) then $x(t)=O\left(e^{-t}\right)$ as $t \rightarrow \infty$, and therefore $a=\lim _{t \rightarrow \infty} \frac{1}{x(t)} e^{-t} \in \mathbb{R}$ exists. It is also easy to see that $z_{i}(t) \rightarrow 0$ as $t \rightarrow \infty$ for $i=1, \ldots, m$. We will show below that such a bounded solution exists.

Solving Equation (4.1) we obtain

$$
x=\frac{1}{a e^{t}+T(-1) \theta(z)}, \text { where } T(b) f(t)=\int_{0}^{\infty} e^{-u} f(t-b u) d u .
$$

The parameter $a$ reflects the invariance of our equation under shifts in the invariant variable $t$. The sign of $a$ is, however, defined uniquely. It must be the same as the sign of

$$
\theta_{0}=\lim _{t \rightarrow \infty} \theta(z)=g_{0}(\omega, \omega)=\frac{1}{\lambda_{0}}\left\langle u_{0}, S\left(u_{0}, u_{0}\right)\right\rangle, \text { where } \omega=(1,0, \ldots, 0) \in \mathbb{R}^{m}
$$

otherwise $x(t)$, with a small $a$, has a singularity for large $t>0$.

LEMMA 4.1. If $\left.\left\langle M_{+} \phi_{\perp}, \phi_{\perp}^{2}\right\rangle_{B-c_{0} I}\right\rangle 0$, where the vector $\phi_{\perp}$ satisfies Equation (2.1), then $\theta_{0}(0)=\lim _{\varepsilon \rightarrow 0} \theta_{0}(\varepsilon)>0$.

The proof of Lemma 4.1 is presented in Section 7.

Remark 4.1. By Assumption 2 and Lemma 4.1, $\theta_{0}(0)=\lim _{\varepsilon \rightarrow 0} \theta_{0}(\varepsilon)$ is positive. Hence, by continuity of $\theta_{0}$ in $\varepsilon$ (see Section 7 ), we can allow $s$ (possibly by choosing it smaller than above) to be such that $\theta_{0}=\theta_{0}(\varepsilon)$ is positive for $0 \leq \varepsilon \leq s$. 
We study only the case $0<\varepsilon \leq s$ below, and therefore we choose $a=1$. Then $x(t)$ must satisfy

$$
x(t)=\frac{1}{e^{t}+T(-1) \theta(z)} .
$$

Furthermore, if the functions $z_{i}=z_{i}(t), i=1, \ldots, m$, are bounded, then they satisfy the integral equations

$$
z_{i}=\mu_{i} T\left(\mu_{i}\right)\left[x\left(g_{i}(Z, Z)-z_{i} \theta(z)\right)\right], i=1, \ldots, m, \text { where } T(b) f(t)=\int_{0}^{\infty} e^{-u} f(t-b u) d u
$$

We denote

$$
g=g(z)=\left(g_{1}, \ldots, g_{m}\right)
$$

We want to prove existence and uniqueness of a solution to the equation

$$
z(t)=\Gamma \Psi(z),
$$

where

$$
\Psi(z)=\frac{1}{e^{t}+T(-1) \theta(z)}[g(z)-z \theta(z)] \text { and } \Gamma=\operatorname{diag}\left(\mu_{1} T\left(\mu_{1}\right), \ldots, \mu_{m} T\left(\mu_{m}\right)\right) .
$$

We denote

$$
\|S\|=\sqrt{\sum_{i=1}^{m} \mathcal{G}_{i}^{2}} \text { and }\|\theta\|=\mathcal{G}_{0}, \text { with } \mathcal{G}_{i}=\sup _{|X|=1}\left|\widehat{g}_{i} X\right|, i=0, \ldots, m .
$$

Then, by Equation (3.10), in the notations of (3.9),

$$
\|S\| \leq \sqrt{m} \frac{C M_{\max } \sigma_{\max }(m+1)}{b_{\min }^{2} \sqrt{M_{\min } \sigma_{\min }}} \text { and }\|\theta\| \leq \frac{C M_{\max } \sigma_{\max }(m+1)}{b_{\min }^{2} \sqrt{M_{\min } \sigma_{\min }}} .
$$

We introduce the Banach space

$$
\mathcal{X}=\left\{z=z(t) \in \mathcal{C}\left(\mathbb{R}, \mathbb{R}^{m}\right) \mid\|z\|<\infty\right\},
$$

where $\mathcal{C}\left(\mathbb{R}, \mathbb{R}^{m}\right)$ denote the space of all continuous bounded functions on $\mathbb{R}$ into $\mathbb{R}^{m}$, and its closed convex subset

$$
\mathcal{B}_{R}=\{z \in \mathcal{X} \mid\|z\| \leq R\}, \text { with } R<R_{*}=\sqrt{1+\frac{\theta_{0}}{\|\theta\|}}-1 \leq \sqrt{2}-1
$$

Furthermore, we introduce the mapping $\mathcal{Z}_{R}: \mathcal{B}_{R} \rightarrow \mathcal{X}$, defined by

$$
\mathcal{Z}_{R}(z)=\left(Z_{1}(z), \ldots, Z_{m}(z)\right), Z_{i}(z)=\mu_{i} T\left(\mu_{i}\right) \frac{g_{i}(Z, Z)-z_{i} \theta(z)}{e^{t}+T(-1) \theta(z)}, i=1, \ldots, m .
$$

Clearly,

$$
|g(z)| \leq\|S\|\left(1+|z|^{2}\right) \text { and }|\theta(z)| \leq\|\theta\|\left(1+|z|^{2}\right)
$$


We note (by bilinearity and symmetry in arguments of $g_{0}$ ) that

$$
\theta(z)=g_{0}\left(\omega+z_{*}, \omega+z_{*}\right)=\theta_{0}+2 g_{0}\left(\omega, z_{*}\right)+g_{0}\left(z_{*}, z_{*}\right),
$$

where

$$
\omega=(1,0, \ldots, 0) \in \mathbb{R}^{m+1} \text { and } z_{*}=\left(0, z_{1}, \ldots, z_{m}\right) .
$$

Also,

$$
\left|g_{0}\left(\omega, z_{*}\right)\right| \leq\|\theta\||z|
$$

and therefore

$$
\theta(z) \geq \theta_{0}-\|\theta\|\left(2\|z\|+\|z\|^{2}\right) .
$$

Hence,

$$
\theta(z) \geq\|\theta\|\left[\left(1+R_{*}\right)^{2}-(1+\|z\|)^{2}\right]
$$

if

$$
\|z\|<R_{*}=\sqrt{1+\frac{\theta_{0}}{\|\theta\|}}-1 .
$$

A similar estimate holds for $T(-1) \theta(z)$ (since $T(b) 1=1$ ), and therefore

$$
\|x\| \leq \frac{1}{\|\theta\|\left[\left(1+R_{*}\right)^{2}-(1+\|z\|)^{2}\right]}, \text { if }\|z\|<R_{*} .
$$

We can now prove the following lemma.

Lemma 4.2. If $z, z^{\prime} \in \mathcal{B}_{R}$, then

$$
\left\|\mathcal{Z}_{R}(z)\right\| \leq \Phi(R) \text { and }\left\|\mathcal{Z}_{R}(z)-\mathcal{Z}_{R}\left(z^{\prime}\right)\right\| \leq \Phi^{\prime}(R)\left\|z-z^{\prime}\right\|,
$$

where

$$
\Phi(R)=\frac{\delta}{\Delta(R)}\left(\frac{\|S\|}{\|\theta\|}+R\right)(1+R)^{2},
$$

with $\delta=\max \left(\left|\mu_{1}\right|, \ldots,\left|\mu_{m}\right|\right)$ and $\Delta(R)=\left(1+R_{*}\right)^{2}-(1+R)^{2}$,

and

$$
\Phi^{\prime}(R)=\frac{d \Phi(R)}{d R}=\frac{1}{\Delta(R)}\left[2 \Phi(R)(1+R)+2 \delta\left(\frac{\|S\|}{\|\theta\|}+R\right)(1+R)+\delta(1+R)^{2}\right]
$$

is the Fréchet derivative of $\Phi(R)$.

Proof. Let $z, z^{\prime} \in \mathcal{B}_{R}$. Then,

$$
\left\|\mathcal{Z}_{R}(z)\right\| \leq \frac{\delta}{\|\theta\| \Delta(R)}(\|g(z)\|+\|z \theta(z)\|) \leq \frac{\delta}{\Delta(R)}\left(\frac{\|S\|}{\|\theta\|}+R\right)(1+R)^{2} .
$$


Clearly,

$$
\begin{aligned}
& \Psi(z)-\Psi\left(z^{\prime}\right) \\
= & \frac{1}{\Delta(z)}[g(z)-z \theta(z)]-\frac{1}{\Delta\left(z^{\prime}\right)}\left[g\left(z^{\prime}\right)-z^{\prime} \theta\left(z^{\prime}\right)\right] \\
= & \frac{1}{\Delta(z)}\left(\left[g(z)-g\left(z^{\prime}\right)\right]+\left(z^{\prime}-z\right) \theta(z)+z^{\prime}\left[\theta\left(z^{\prime}\right)-\theta(z)\right]+\left[\Delta\left(z^{\prime}\right)-\Delta(z)\right] \Psi\left(z^{\prime}\right)\right),
\end{aligned}
$$

where

$$
\Psi(z)=\frac{1}{e^{t}+T(-1) \theta(z)}[g(z)-z \theta(z)] \text { and } \Delta(z)=e^{t}+T(-1) \theta(z) .
$$

We note (by bilinearity and symmetry in arguments of $g_{i}$ for $i=0, \ldots, m$ ) that

$$
\begin{aligned}
g_{i}(Z, Z)-g_{i}\left(Z^{\prime}, Z^{\prime}\right) & =g_{i}\left(Z-Z^{\prime}, Z+Z^{\prime}\right) \text { for } i=0, \ldots, m, \\
\text { where } Z & =\omega+z_{*}=\left(1, z_{1}, \ldots, z_{m}\right) .
\end{aligned}
$$

Therefore

$$
\left\|\theta\left(z^{\prime}\right)-\theta(z)\right\| \leq 2\|\theta\|(1+R)\left\|z-z^{\prime}\right\|
$$

and

$$
\left\|g(z)-g\left(z^{\prime}\right)\right\| \leq 2\|S\|(1+R)\left\|z-z^{\prime}\right\| .
$$

Hence,

$$
\begin{gathered}
\left\|\mathcal{Z}_{R}(z)-\mathcal{Z}_{R}\left(z^{\prime}\right)\right\| \leq \delta\left\|\Psi(z)-\Psi\left(z^{\prime}\right)\right\| \\
\leq \frac{1}{\Delta(R)}\left[2 \Phi(R)(1+R)+2 \delta\left(\frac{\|S\|}{\|\theta\|}+R\right)(1+R)+\delta(1+R)^{2}\right]\left\|z-z^{\prime}\right\| .
\end{gathered}
$$

Let us now consider the equation

$$
r=\Phi(r), r \in I=\left[0, R_{*}\right), \Phi(r)=\frac{\delta}{\Delta(r)}\left(\frac{\|S\|}{\|\theta\|}+r\right)(1+r)^{2} .
$$

Clearly,

$$
\Phi(r)>0, \Phi^{\prime}(r)>0, \Phi^{\prime \prime}(r)>0, \text { for all } r \in I .
$$

Then there are three different possibilities:

1. Equation (4.5) has exactly two different solutions $r=r_{1}$ and $r=r_{2}, r_{1}<r_{2}$, and there exists a unique point $r=r_{0}, r_{1}<r_{0}<r_{2}$, such that $\Phi^{\prime}\left(r_{0}\right)=1$;

2. Equation (4.5) has a unique solution $r=r_{1}$, and $\Phi^{\prime}\left(r_{1}\right)=1\left(r_{1}=r_{2}=r_{0}\right)$;

3. Equation (4.5) has no solutions.

We consider the first case. Obviously, $\mathcal{Z}_{R}: \mathcal{B}_{R} \rightarrow \mathcal{B}_{R}$ for all $R \in\left[r_{1}, r_{2}\right]$. Moreover, if $R \in\left[r_{1}, r_{0}\right)$ then $\mathcal{Z}_{R}$ is a contraction, since $\Phi^{\prime}(R)<1$. We can state the following theorem.

Theorem 4.3. Assume that the equation

$$
r=\Phi(r), r \in I=\left[0, R_{*}\right),
$$


has two different solutions $r_{1}$ and $r_{2}, r_{1}<r_{2}$, in $I$, and let $r_{0}$ be the unique point such that $r_{1}<r_{0}<r_{2}$ and $\Phi^{\prime}\left(r_{0}\right)=1$.

Then the mapping $\mathcal{Z}_{R}(z): \mathcal{B}_{R} \rightarrow \mathcal{X}, R \in\left[r_{1}, R_{*}\right)$, has a fixed point $z=z^{*}$.

The fixed point $z=z^{*}$ is unique in the ball

$$
0 \leq\|z\|<r_{2}, z \in \mathcal{B}_{R}
$$

and satisfies the inequality

$$
\left\|z^{*}\right\| \leq r_{1}
$$

Furthermore, the iteration process

$$
z_{n+1}=\mathcal{Z}_{R}\left(z_{n}\right), n=0,1, \ldots
$$

converges to $z^{*}$ for any $z_{0} \in \mathcal{B}_{R}$ such that $\left\|z_{0}\right\|<r_{0}$.

Proof. The mapping $\mathcal{Z}_{r}(z): \mathcal{B}_{r} \rightarrow \mathcal{B}_{r}$ is a contraction for all $r_{1} \leq r<r_{0}$, and therefore $\mathcal{Z}_{R}(z), R \in\left[r_{1}, R_{*}\right)$, has a unique fixed point $z=z^{*}$ in $\mathcal{B}_{r_{1}}$, and the iteration process converges to $z^{*}$ for any $z_{0} \in \mathcal{B}_{R}$ such that $\left\|z_{0}\right\|<r_{0}$.

If $z=z^{* *}$ is a fixed point of the mapping $\mathcal{Z}_{R}(z)$ and $\left\|z^{* *}\right\| \in\left(r_{1}, r_{2}\right)$, then $\left\|z^{* *}\right\|=$ $\left\|\mathcal{Z}_{R}\left(z^{* *}\right)\right\| \leq \Phi\left(\left\|z^{* *}\right\|\right)<\left\|z^{* *}\right\|$. Contradiction.

Therefore, uniqueness in $\mathcal{B}_{r_{1}}$ implies uniqueness in the ball $0 \leq\|z\|<r_{2}, z \in \mathcal{B}_{R}$.

REMARK 4.2. In fact, according to [11], the iteration process

$$
z_{n+1}=\mathcal{Z}_{R}\left(z_{n}\right), n=0,1, \ldots
$$

converges to $z^{*}$ for any $z_{0} \in \mathcal{B}_{R}$ such that $\left\|z_{0}\right\|<r_{2}$.

REMARK 4.3. Let the equation

$$
r=\Phi(r), r \in I=\left[0, R_{*}\right)
$$

have a unique solution $r=r_{1}$, and $\Phi^{\prime}\left(r_{1}\right)=1$. Then according to [11], $\mathcal{Z}_{R}(z), R \in$ $\left[r_{1}, R_{*}\right)$, has a unique fixed point $z=z^{*}$ in $\mathcal{B}_{r_{1}}$.

COROLlary 4.4. There exists a function

$$
\delta_{0}=\delta_{0}\left(R_{*}, \frac{\|S\|}{\|\theta\|}\right)
$$

such that the condition

$$
\delta<\delta_{0}
$$

is sufficient for the existence and uniqueness of the fixed point $z=z^{*}$ for the mapping $\mathcal{Z}_{R}(z)$.

Proof. Let $\delta_{0}=\delta_{0}\left(R_{*}, \frac{\|S\|}{\|\theta\|}\right)$ be the value of $\delta$, such that $r_{0}=r_{1}=r_{2}$. 


\section{Proof of Theorem 3.1}

Proof. Let $s$ be a non-zero number such that, with $c=c_{0}+\varepsilon$,

$$
0<s<\min _{\xi_{i}^{1}>c_{0}}\left(\xi_{i}^{1}-c_{0}\right)
$$

or, equivalently,

$$
\operatorname{det}(B-c I) \neq 0, \text { if } \varepsilon \in[0, s]
$$

and

$$
\begin{gathered}
\operatorname{det}\left(\left\langle M_{+} \phi_{i}, \phi_{j}\right\rangle_{B-c I}\right) \neq 0 \text { for all } \varepsilon \in(0, s], \\
\theta_{0}(\varepsilon)>0 \text { for all } \varepsilon \in[0, s],
\end{gathered}
$$

where $\theta_{0}(\varepsilon)=\frac{\left\langle u_{0}, S\left(u_{0}, u_{0}\right)\right\rangle}{\lambda_{0}}$ if $\varepsilon>0$ and $\theta_{0}(0)=\lim _{\varepsilon \rightarrow 0^{+}} \theta_{0}(\varepsilon)$.

Such a number $s$ exists, by Assumption 1 and Remark 4.1.

We construct the function

$$
\Phi_{s}(R)=\delta \frac{\left(\frac{\|S\|_{s}}{\|\theta\|_{s}}+R\right)(1+R)^{2}}{\left(1+R_{* s}\right)^{2}-(1+R)^{2}},
$$

where

$$
\delta=\delta(\varepsilon)=\max \left(\left|\mu_{1}\right|, \ldots,\left|\mu_{m}\right|\right) \text { and } R_{* s}=\sqrt{1+\frac{\theta_{0, s}}{\|\theta\|_{s}}}-1 \leq \sqrt{2}-1
$$

Here

$$
\|S\|_{s}=\max _{0 \leq \varepsilon \leq s}(\|S\|),\|\theta\|_{s}=\max _{0 \leq \varepsilon \leq s}(\|\theta\|) \text { and } \theta_{0, s}=\min _{0 \leq \varepsilon \leq s}\left(\theta_{0}\right)>0,
$$

such that

$$
\left|g_{0}(Z, Z)\right| \leq\|\theta\||Z|^{2} \text { and }|g(Z, Z)| \leq\|S\||Z|^{2} \text {, with } g=\left(g_{1}, \ldots, g_{m}\right) .
$$

Then, by Equation (4.2), in the notations (3.9) and with $b_{s}=\min _{0 \leq \varepsilon \leq s, 0 \leq \alpha \leq n}\left|\xi_{\alpha}^{1}-c\right|$,

$$
\|S\|_{s} \leq \sqrt{m} \frac{C M_{\max } \sigma_{\max }(m+1)}{b_{s}^{2} \sqrt{M_{\min } \sigma_{\min }}} \text { and }\|\theta\|_{s} \leq \frac{C M_{\max } \sigma_{\max }(m+1)}{b_{s}^{2} \sqrt{M_{\min } \sigma_{\min }}} .
$$

One can show that

$$
0 \leq \delta \frac{\left(\frac{\|S\|}{\|\theta\|}+R\right)(1+R)^{2}}{\left(1+R_{*}\right)^{2}-(1+R)^{2}} \leq \Phi_{s}(R), \text { for } \varepsilon \in[0, s] .
$$

Let $\delta_{0}=\delta_{0}\left(R_{* s}, \frac{\|S\|_{s}}{\|\theta\|_{s}}\right)$ be the value of $\delta$, such that the equation

$$
r=\Phi_{s}(r), r \in\left[0, R_{* s}\right)
$$


has a unique solution $R_{s}$. By the relations (3.8), $\delta \rightarrow 0$ if $\varepsilon \rightarrow 0$. Hence, there exists a non-zero number $0<s_{0} \leq s$, such that $\delta<\delta_{0}$ if $0 \leq \varepsilon \leq s_{0}$.

Let $z^{*}=\left(z_{1}^{*}, \ldots, z_{m}^{*}\right)$ be a solution of Equation (3.12); then

$$
F(y)=M_{+}^{1 / 2}\left[M_{+}^{1 / 2}+\lambda_{0} x(t) U Z^{*}(t)\right]
$$

where $U$ is the matrix with columns $u_{0}, \ldots, u_{m}$, and $Z^{*}=\left(1, z_{1}^{*}, \ldots, z_{m}^{*}\right)$. Furthermore,

$$
M_{\min }=\min _{1 \leq i \leq n}\left(M_{+i}\right)>0, \text { where } M_{+}=\left(M_{+1}, \ldots, M_{+n}\right),
$$

and

$$
\left\|\lambda_{0} x(t) U Z^{*}(t)\right\| \leq \lambda_{0} \frac{\sqrt{1+\left\|R_{s}\right\|^{2}} \sqrt{\sigma_{\max }}}{\|\theta\|_{s} b_{s}\left[\left(1+R_{* s}\right)^{2}-\left(1+\left\|R_{s}\right\|\right)^{2}\right]} \leq M_{\min }^{1 / 2}
$$

if $\varepsilon$ is sufficiently small, since $\frac{\sqrt{1+\left\|R_{s}\right\|^{2}} \sqrt{\sigma_{\max }}}{\|\theta\|_{s} b_{s}\left[\left(1+R_{* s}\right)^{2}-\left(1+\left\|R_{s}\right\|\right)^{2}\right]}$ is independent of $\varepsilon$ and $\lambda_{0} \rightarrow 0$ as $\varepsilon \rightarrow 0$.

Hence, $F(y) \geq 0$ if $\varepsilon$ is sufficiently small, and the theorem is proved.

6. Convergence to a Maxwellian as $y \rightarrow-\infty$

In the continuous case there is at most one more Maxwellian $M$, besides $M_{+}$, that fulfills the relations

$$
\left\langle M, \phi_{i}\right\rangle_{B-c I}=\left\langle M_{+}, \phi_{i}\right\rangle_{B-c I}, i=1, \ldots, p .
$$

For DVMs, we will see that for sufficiently small $\varepsilon>0$ and (at least) in a neighborhood of $M_{+}$, there is exactly one more Maxwellian $M$, besides $M_{+}$, that fulfills the relations (6.1).

Lemma 6.1. Let $\delta<\delta_{0}$, where $\delta=\max \left(\left|\mu_{1}\right|, \ldots,\left|\mu_{m}\right|\right)$ and $\delta_{0}=\delta_{0}\left(R_{*}, \frac{\|S\|}{\|\theta\|}\right)$ is the function defined by Corollary 4.4. Then Equation (3.11) have a unique non-trivial stationary solution, such that $z \in \mathcal{B}_{R}$ (4.3).

Furthermore, the solution can be obtained by the iteration process

$$
z_{n+1}=\mathcal{Z}_{0 R}\left(z_{n}\right), n=0,1, \ldots,
$$

if $\left\|z_{0}\right\|$ is sufficiently small (cf. Theorem 4.3).

Proof. Consider Equation (3.11) for the stationary case, i.e.,

$$
\left\{\begin{array}{l}
x=x^{2} \theta(z) \\
\frac{\lambda_{i}}{\lambda_{0}} x z_{i}=x^{2} g_{i}(Z, Z),
\end{array}\right.
$$

$x=0$ in Equation (6.2) corresponds to the trivial stationary solution $h=0$, or $F=$ $M_{+}$in the original notation. Hence, we assume that $x \neq 0$ and obtain the algebraic equations

$$
\left\{\begin{array}{l}
x=\frac{1}{\theta(z)} \\
z_{i}=\mu_{i}\left(\frac{g_{i}(Z, Z)}{\theta(z)}-z_{i}\right),
\end{array} \mu_{i}=\frac{\lambda_{0}}{\lambda_{i}-\lambda_{0}}, i=1, \ldots, m\right.
$$


We define a mapping $\mathcal{Z}_{0 R}: \mathcal{B}_{R} \rightarrow \mathcal{X}$ by

$$
\mathcal{Z}_{0 R}(z)=\left(Z_{01}(z), \ldots, Z_{0 m}(z)\right), Z_{0 i}(z)=\mu_{i}\left(\frac{g_{i}(Z, Z)}{\theta(z)}-z_{i}\right), i=1, \ldots, m .
$$

Let $z, z^{\prime} \in \mathcal{B}_{R}$. Then

$$
\left\|\mathcal{Z}_{0 R}(z)\right\| \leq \delta\left(\frac{\|g(z)\|}{\|\theta\| \Delta(R)}+\|z\|\right) \leq \delta\left(\frac{\|S\|}{\|\theta\|} \frac{(1+R)^{2}}{\Delta(R)}+R\right) \leq \Phi(R)
$$

and

$$
\begin{gathered}
\left\|\mathcal{Z}_{0 R}(z)-\mathcal{Z}_{0 R}\left(z^{\prime}\right)\right\| \leq \delta\left\|\frac{g(z)-g\left(z^{\prime}\right)}{\theta(z)}-g\left(z^{\prime}\right) \frac{\theta(z)-\theta\left(z^{\prime}\right)}{\theta\left(z^{\prime}\right) \theta(z)}+z-z^{\prime}\right\| \leq \\
\leq \delta\left[2 \frac{\|S\|}{\|\theta\|} \frac{1+R}{\Delta(R)}\left(1+\frac{(1+R)^{2}}{\Delta(R)}\right)+1\right]\left\|z-z^{\prime}\right\| \leq \Phi^{\prime}(R)\left\|z-z^{\prime}\right\|
\end{gathered}
$$

in the notations of Section 4.

Now we can apply corresponding results to Theorem 4.3 and Corollary 4.4 for $\mathcal{Z}_{0 R}(z)$ (instead of $\mathcal{Z}_{R}(z)$ ) and the lemma is proved.

Corollary 6.2. Let $\left\{u_{0}, \ldots, u_{m}\right\}$ be the basis (3.6) of $\operatorname{Im}\left((B-c I)^{-1} L\right)$ and let $R_{s}$ be chosen as in the proof of Theorem 3.1 in Section 5. Then there exists a unique Maxwellian of the form

$$
M_{-}=M_{+}+M_{+}^{1 / 2} \lambda_{0} x\left(u_{0}+\sum_{i=1}^{m} z_{i} u_{i}\right), x \neq 0, z=\left(z_{1}, \ldots, z_{m}\right) \in B_{R_{s}},
$$

provided that $\varepsilon>0$ is sufficiently small. Furthermore, $M_{-}$fulfills Equation (6.1).

Proof. Every positive vector of the form (6.4), where $\left(x, z_{1}, \ldots, z_{m}\right)$ is a non-trivial stationary solution of Equation (3.11), is a Maxwellian. We choose $\left(x, z_{1}, \ldots, z_{m}\right)$ as the solution in Lemma 6.1 for $R=R_{s}$, and note that $M_{-}$is positive (cf. the proof of Theorem 3.1 in Section 5) and therefore also a Maxwellian, provided that $\varepsilon>0$ is sufficiently small. The uniqueness follows from the uniqueness in Lemma 6.1, since every Maxwellian $M_{-}$of the form (6.4) corresponds to a non-trivial stationary solution of Equation (3.11).

The last statement follows by the relations

$$
\left\langle u_{i}, M_{+}^{1 / 2} \phi_{i}\right\rangle_{B-c I}=0, \text { for } i=0, \ldots, m \text {. }
$$

Now we prove Theorem 2.1.

Proof. (of Theorem 2.1) We apply a method used in [7]. Let $F$ be the locally unique non-negative solution in Theorem 3.1. We define

$$
H[F]=H[F](y)=\sum_{i=1}^{n} \xi_{i}^{1} \mu\left(F_{i}(y)\right)
$$

where

$$
\mu(x)=\left\{\begin{array}{c}
x \log x \text { if } x>0 \\
0 \text { if } x=0
\end{array}\right.
$$


It is a well-known fact (multiply Equation (3.1) by $1+\log F$ ) that

$$
\frac{d}{d y} H[F]=\frac{1}{4} \sum_{i, j, k, l=1}^{n}\left[\Gamma_{i j}^{k l}\left(F_{k} F_{l}-F_{i} F_{j}\right) \log \frac{F_{i} F_{j}}{F_{k} F_{l}}\right] \leq 0,
$$

with equality if, and only if, $F_{k} F_{l}=F_{i} F_{j}$ for all indices $1 \leq i, j, k, l \leq n$ such that $\Gamma_{i j}^{k l} \neq 0$. That is, the inequality (6.5) is an equality, if, and only if, $F$ is a Maxwellian.

The function $F$ is bounded, and so the derivative $\frac{d F}{d y}$ and $H[F]$ are also bounded. Hence, $H[F](-\infty):=\lim _{y \rightarrow-\infty} H[F]$ exists and is finite. Consequently,

$$
\int_{-\infty}^{0} \frac{d}{d y} H[F] d y=H[F](0)-H[F](-\infty)
$$

is a finite non-positive number.

We denote by $\mathcal{M}$ the set of all Maxwellians fulfilling the relations (6.1). We want to prove that

$$
\operatorname{dist}\left(F\left(y_{\nu}\right), \mathcal{M}\right) \rightarrow 0 \text { as } \nu \rightarrow \infty
$$

for any decreasing sequence $\left\{y_{\nu}\right\}_{\nu=1}^{\infty}$ of negative real numbers, such that $y_{\nu} \rightarrow-\infty$ as $\nu \rightarrow \infty$. We suppose the opposite. Then there are positive numbers $\epsilon_{1}>0$ and $\delta_{1}>0$, and a decreasing sequence $\left\{t_{\nu}\right\}_{\nu=1}^{\infty}$ of negative real numbers, such that $\left|t_{\nu}-t_{\nu+1}\right| \geq \epsilon_{1}$ and $\operatorname{dist}\left(F\left(t_{\nu}\right), \mathcal{M}\right) \geq \delta_{1}$. The derivative of $F$ is bounded on $\mathbb{R}$, and therefore there is a positive number $\epsilon_{2}>0$, such that $\epsilon_{2}<\frac{\epsilon_{1}}{2}$ and $\operatorname{dist}(F(t), \mathcal{M}) \geq \frac{\delta_{1}}{2}$, if $t \in J_{\nu}=\left[t_{\nu}-\right.$ $\left.\epsilon_{2}, t_{\nu}+\epsilon_{2}\right]$ and $\nu \in\{1,2, \ldots\}$.

We denote

$$
\Psi\left(J_{\nu}\right)=-\int_{t_{\nu}-\epsilon_{2}}^{t_{\nu}+\epsilon_{2}} \frac{d}{d y} H[F](y) d y, \nu=1,2, \ldots,
$$

and recall that for each $\nu$ there exists a number $s_{\nu} \in J_{\nu}$, such that

$$
\int_{t_{\nu}-\epsilon_{2}}^{t_{\nu}+\epsilon_{2}} \frac{d}{d y} H[F](y) d y=2 \epsilon_{2} \frac{d}{d y} H[F]\left(s_{\nu}\right) .
$$

Hence, the terms $\Psi\left(J_{\nu}\right) \rightarrow 0$ as $\nu \rightarrow \infty$, if, and only if, $\frac{d}{d y} H[F]\left(s_{\nu}\right) \rightarrow 0$ as $\nu \rightarrow \infty$.

The sequence $\left\{F\left(s_{\nu}\right)\right\}_{\nu=1}^{\infty}$ is bounded, and hence, by the Bolzano-Weierstrass theorem, we can extract a subsequence $\left\{F\left(s_{\alpha}\right)\right\}_{\alpha=1}^{\infty}$ such that $\lim _{\alpha \rightarrow \infty} F\left(s_{\alpha}\right)=N$ exists. Clearly, $\Psi\left(J_{\alpha}\right)$ is non-negative for all $\alpha$, and the series

$$
\sum_{\alpha=1}^{\infty} \Psi\left(J_{\alpha}\right) \leq-\int_{-\infty}^{0} \frac{d}{d y} H[F] d y
$$


converges. Hence, $\frac{d}{d y} H[F]\left(s_{\alpha}\right) \rightarrow 0$ as $\alpha \rightarrow \infty$. We obtain (since $F$ is continuous)

$$
\frac{1}{4} \sum_{i, j, k, l=1}^{n}\left[\Gamma_{i j}^{k l}\left(N_{k} N_{l}-N_{i} N_{j}\right) \log \frac{N_{i} N_{j}}{N_{k} N_{l}}\right]=\lim _{\alpha \rightarrow \infty} \frac{d}{d y} H[F]\left(s_{\alpha}\right)=0,
$$

and so $N$ must be a Maxwellian. This is a contradiction, since $\operatorname{dist}\left(F\left(s_{\alpha}\right), \mathcal{M}\right) \geq \frac{\delta_{1}}{2}$ for all $\alpha$. Hence,

$$
\operatorname{dist}(F(y), \mathcal{M}) \rightarrow 0 \text { as } y \rightarrow-\infty .
$$

By the construction of the solution $F$ in Theorem 3.1, it is clear, by Corollary 6.2, that $F(y)$ must converge to the Maxwellian $M_{-}$of Corollary 6.2 as $y \rightarrow-\infty$.

\section{Proof of Lemma 4.1}

Proof. There is a unique vector function $\psi=\psi(\varepsilon)$, such that

$$
L \psi=\lambda_{0}(B-c I) \psi, \psi(0)=\psi_{0}=M^{1 / 2} \phi_{\perp} \text { and }\left\langle\psi, \psi_{0}\right\rangle=1(0 \leq \varepsilon \leq s),
$$

where $\lambda_{0}=\lambda_{0}(\varepsilon) \geq 0$ with equality if, and only if, $\varepsilon=0$. By Remark 3.2, the eigenvalue $\lambda_{0}=\lambda_{0}(\varepsilon)$ is a $C^{1}$-function (in an open neighborhood of $\varepsilon=0$ ). Let $\left\{e_{1}, \ldots, e_{p-1}, e_{p}=\psi_{0}\right\}$ be a basis of $N(L)$, such that Equation (3.3), (3.4) are fulfilled. Then

$$
\psi(\varepsilon)=\psi_{0}+\psi^{\perp}(\varepsilon)+\sum_{\alpha=1}^{p-1} \rho_{\alpha}(\varepsilon) e_{\alpha}
$$

for $\quad$ some functions $\quad \rho_{1}, \ldots, \rho_{p-1}:[0, s] \rightarrow \mathbb{R} \quad$ and $\quad \psi^{\perp}:[0, s] \rightarrow \operatorname{Im}(L)=N(L)^{\perp}=$ $\left\{x \in \mathbb{R}^{n} \mid\langle x, y\rangle=0\right.$ for all $\left.y \in N(L)\right\}$. By Equation (7.1),

$$
\lambda_{0}\left[\left\langle\psi^{\perp}, e_{\alpha}\right\rangle_{B}+\rho_{\alpha}\left\langle e_{\alpha}, e_{\alpha}\right\rangle_{B-c I}\right]=\left\langle L \psi, e_{\alpha}\right\rangle=0, \alpha=1, \ldots, p-1,
$$

or, if $\varepsilon \neq 0$, equivalently,

$$
\rho_{\alpha}=\frac{\left\langle\psi^{\perp}, e_{\alpha}\right\rangle_{B}}{\left\langle e_{\alpha}, e_{\alpha}\right\rangle_{B-c I}}, \alpha=1, \ldots, p-1
$$

But, $\psi^{\perp} \rightarrow 0$ as $\varepsilon \rightarrow 0$, since $L \psi^{\perp}=L \psi=\lambda_{0}(B-c I) \psi \rightarrow 0$ as $\varepsilon \rightarrow 0$. Hence, $\rho_{\alpha} \rightarrow 0$ as $\varepsilon \rightarrow 0$. Then $\psi^{\perp}$ is differentiable at $\varepsilon=0$, since

$$
\frac{d \psi^{\perp}}{d \varepsilon}(0)=\lim _{\varepsilon \rightarrow 0} \frac{\psi^{\perp}(\varepsilon)}{\varepsilon}=\lim _{\varepsilon \rightarrow 0}\left(\frac{\lambda_{0}}{\varepsilon} L^{-1}(B-c I) \psi(\varepsilon)\right)=\lambda_{0}^{\prime} L^{-1}\left(B-c_{0} I\right) \psi_{0}
$$

(where $L^{-1}: \operatorname{Im}(L) \rightarrow \operatorname{Im}(L)$ is defined in a natural way) exists. Here and below, we denote $\lambda_{0}^{\prime}=\frac{d \lambda_{0}}{d \varepsilon}(0)$ and $\varphi_{0}^{\prime}=\frac{d \psi^{\perp}}{d \varepsilon}(0)$. Then,

$$
L \varphi_{0}^{\prime}=\lambda_{0}^{\prime}\left(B-c_{0} I\right) \psi_{0}
$$

Clearly,

$$
\theta_{0}(\varepsilon)=\frac{1}{\lambda_{0}}\left\langle u_{0}, S\left(u_{0}, u_{0}\right)\right\rangle=q^{3} \frac{\langle\psi, S(\psi, \psi)\rangle}{\lambda_{0}}, \text { where } q=\left\langle u_{0}, \psi_{0}\right\rangle_{B-c_{0} I} .
$$


Moreover, $Q\left(M e^{\theta \phi_{\perp}}, M e^{\theta \phi_{\perp}}\right)=0$ for all $\theta \in \mathbb{R}$. Considering the terms of order $O\left(\theta^{2}\right)$ as $\theta \rightarrow 0$, we obtain that $Q\left(M \phi_{\perp}^{2}, M\right)=-Q\left(M \phi_{\perp}, M \phi_{\perp}\right)$. Hence,

$$
S\left(\psi_{0}, \psi_{0}\right)=M^{-1 / 2} Q\left(M \phi_{\perp}, M \phi_{\perp}\right)=-M^{-1 / 2} Q\left(M \phi_{\perp}^{2}, M\right)=\frac{1}{2} L\left(M^{1 / 2} \phi_{\perp}^{2}\right) .
$$

Finally, we conclude that

$$
\begin{aligned}
\theta_{0}(0) & =\lim _{\varepsilon \rightarrow 0^{+}} \theta_{0}(\varepsilon)=\frac{q^{3}}{\lambda_{0}^{\prime}}\left\langle\varphi_{0}^{\prime}, S\left(\psi_{0}, \psi_{0}\right)\right\rangle=\frac{q^{3}}{2 \lambda_{0}^{\prime}}\left\langle L \varphi_{0}^{\prime}, M^{1 / 2} \phi_{\perp}^{2}\right\rangle \\
& =\frac{q^{3}}{2}\left\langle M \phi_{\perp}, \phi_{\perp}^{2}\right\rangle_{B-c_{0} I}>0,
\end{aligned}
$$

if $\left\langle M \phi_{\perp}, \phi_{\perp}^{2}\right\rangle_{B-c_{0} I}>0$ and $q=\left\langle u_{0}, \psi_{0}\right\rangle=\left\langle u_{0}, M^{1 / 2} \phi_{\perp}\right\rangle>0$. The lemma is proved.

Acknowledgement. The work was supported by the Swedish Research Council (grants 2003-5357 and 2006-3404).

\section{REFERENCES}

[1] V.I. Arnold, Geometrical Methods in the Theory of Ordinary Differential Equations, SpringerVerlag, 2nd ed., 1988.

[2] N. Bernhoff, On Half-Space and Shock-Wave Problems for Discrete Velocity Models of the Boltzmann Equation, Karlstad University Studies, Ph.D thesis, 2005.

[3] A.V. Bobylev and N. Bernhoff, Discrete velocity models and dynamical systems, Lecture Notes on the Discretization of the Boltzmann Equation, N. Bellomo and R. Gatignol, eds., World Scientific, 203-222, 2003.

[4] C. Bose, R. Illner and S. Ukai, On shock wave solutions for discrete velocity models of the Boltzmann equation, Transp. Th. Stat. Phys., 27, 35-66, 1998.

[5] H. Cabannes, The discrete Boltzmann equation, 1980 (2003). Lecture notes given at the University of California at Berkeley, 1980, revised with R. Gatignol and L.-S. Luo, 2003.

[6] R.E. Caflisch and B. Nicolaenko, Shock profile solutions of the Boltzmann equation, Comm. Math. Phys., 86, 161-194, 1982.

[7] C. Cercignani, R. Illner, M. Pulvirenti and M. Shinbrot, On nonlinear stationary half-space problems in discrete kinetic theory, J. Stat. Phys., 52, 885-896, 1988.

[8] F. Coron, F. Golse and C. Sulem, Classification of well-posed kinetic layer problems, Comm. Pure Appl. Math., 41, 409-435, 1988.

[9] A. Dressel and W.A. Yong, Existence of traveling-wave solutions for hyperbolic systems of balance laws, Arch. Ration. Mech. Anal., 182, 49-75, 2006.

[10] R. Gatignol, Théorie Cinétique des Gaz Répartition Discrète de Vitesses, Springer-Verlag, 1975.

[11] L.V. Kantorovich and G.P. Akilov, Functional Analysis in Normed Spaces, Pergamon Press, 1964.

[12] T. Kato, Perturbation Theory for Linear Operators, Springer-Verlag, 1995.

[13] T.P. Liu and S.H. Yu, Boltzmann equation: micro-macro decompositions and positivity of shock profiles, Comm. Math. Phys., 246, 133-179, 2004. 